\title{
Redshifted 21 Centimeter Signatures around the Highest Redshift Quasars
}

\section{Citation}

Wyithe, J. Stuart B., and Abraham Loeb. 2004. "Redshifted 21 Centimeter Signatures around the Highest Redshift Quasars." The Astrophysical Journal 610 (1): 117-27. https:// doi.org/10.1086/421042.

\section{Permanent link}

http://nrs.harvard.edu/urn-3:HUL.InstRepos:41393393

\section{Terms of Use}

This article was downloaded from Harvard University's DASH repository, and is made available under the terms and conditions applicable to Other Posted Material, as set forth at http:// nrs.harvard.edu/urn-3:HUL.InstRepos:dash.current.terms-of-use\#LAA

\section{Share Your Story}

The Harvard community has made this article openly available.

Please share how this access benefits you. Submit a story.

Accessibility 


\title{
REDSHIFTED 21 CENTIMETER SIGNATURES AROUND THE HIGHEST REDSHIFT QUASARS
}

\author{
J. Stuart B. Wyithe ${ }^{1}$ and Abraham Loeb ${ }^{2}$ \\ Received 2004 January 26; accepted 2004 March 16
}

\begin{abstract}
The Ly $\alpha$ absorption spectrum of the highest redshift quasars indicates that they are surrounded by giant $\mathrm{H}$ II regions, a few megaparsecs in size. The neutral gas around these $\mathrm{H}$ II regions should emit $21 \mathrm{~cm}$ radiation in excess of the cosmic microwave background and enable future radio telescopes to measure the transverse extent of these $\mathrm{H}$ II regions. At early times, the $\mathrm{H}$ II regions expand with a relativistic speed. Consequently, their measured sizes along the line of sight (via Ly $\alpha$ absorption) and transverse to it (via $21 \mathrm{~cm}$ emission) should have different observed values due to relativistic time delay. We show that the combined measurement of these sizes would directly constrain the neutral fraction of the surrounding intergalactic medium (IGM) as well as the quasar lifetime. Based on current number counts of luminous quasars at $z \gtrsim 6$, an instrument like LOFAR should detect $\gtrsim 2$ redshifted $21 \mathrm{~cm}$ shells per field (with a radius of $11^{\circ}$ ) around active quasars as bright as those already discovered by Sloan Digital Sky Survey, and $\gtrsim 200$ relic shells of inactive quasars per field. We show that Ly $\alpha$ photons from the quasar are unable to heat the IGM or to couple the spin and kinetic temperatures of atomic hydrogen beyond the edge of the $\mathrm{H}$ II region. The detection of the IGM in $21 \mathrm{~cm}$ emission around high-redshift quasars would therefore gauge the presence of a cosmic Ly $\alpha$ background during the reionization epoch.
\end{abstract}

Subject headings: cosmology: theory — intergalactic medium — quasars: general — radio lines: general

\section{INTRODUCTION}

The recent discovery of quasars at $z>6$ (Fan et al. 2001, 2003 ) and the subsequent observation of two Gunn-Peterson (1965) troughs (White et al. 2003) established a new era in studies of the end of the reionization epoch. The absorption of Ly $\alpha$ photons from these quasars probes the ionization state of the hydrogen in the intergalactic medium (IGM) near the quasar redshift and has revealed giant ionized regions (Cen \& Haiman 2000; Madau \& Rees 2000). Comparison of the radii of these regions with the ionizing flux implies quasar ages of $\lesssim 10^{7} \mathrm{yr}$ and an ionization front that is expanding with a relativistic speed (Wyithe \& Loeb 2004).

The generation of planned low-frequency instruments (such as $\operatorname{LOFAR}^{3}$ or the Square Kilometer Array telescope ${ }^{4}$ ) will open a new window on the state of hydrogen in the prereionization universe through the emission or absorption of $21 \mathrm{~cm}$ photons relative to the cosmic microwave background (CMB) by neutral hydrogen in the IGM (Scott \& Rees 1990; Madau et al. 1997; Gnedin \& Ostriker 1997; Shaver et al. 1999; Tozzi et al. 2000; Iliev et al. 2002, 2003; Ciardi \& Madau 2003; Furlanetto et al. 2004; Zaldarriaga et al. 2004; Gnedin \& Shaver 2003; Loeb \& Zaldarriaga 2003). The giant $\mathrm{H}$ II regions surrounding the highest redshift quasars should be visible via rings of redshifted $21 \mathrm{~cm}$ emission from warm but neutral gas beyond the ionization front.

The relativistic expansion of the $\mathrm{H}$ II region implies that the radii measured along and transverse to the line of sight using the $\mathrm{Ly} \alpha$ and $21 \mathrm{~cm}$ techniques, respectively, probe different epochs as a result of relativistic time delay. In this paper we discuss what will be learned about the state of the IGM surrounding the highest redshift by combining Ly $\alpha$ and $21 \mathrm{~cm}$

\footnotetext{
${ }^{1}$ University of Melbourne, Parkville, Victoria, Australia; swyithe@isis.ph unimelb.edu.au.

${ }^{2}$ Harvard-Smithsonian Center for Astrophysics, 60 Garden Street, Cambridge, MA 02138; aloeb@cfa.harvard.edu.

3 See http://www.lofar.org.

${ }^{4}$ See http://www.skatelescope.org.
}

measurements. In $\S 2$ we discuss the measurements of radii of the $\mathrm{H}$ II region in Ly $\alpha$ absorption and redshifted $21 \mathrm{~cm}$ emission. The relation between these radii and the neutral fraction of the IGM are then discussed in $\S 3$. Some examples of the brightness temperature profiles around high-redshift quasars are presented in $\S 4$. In $\S 5$ we discuss the observation of fossil $\mathrm{H}$ II regions and their utility as a probe of the radiation background at high redshift. Finally, in $\S 6$ we discuss the utility of quasar Strömgren spheres in the calibration of the global signature of reionization. Section 7 summarizes our main results. Throughout the paper we adopt the set of cosmological parameters determined by the Wilkinson Microwave Anisotropy Probe (WMAP; Spergel et al. 2003), namely, mass density parameters of $\Omega_{m}=0.27$ in matter, $\Omega_{b}=0.044$ in baryons, $\Omega_{\Lambda}=0.73$ in a cosmological constant, and a Hubble constant of $H_{0}=71 \mathrm{~km} \mathrm{~s}^{-1} \mathrm{Mpc}^{-1}$.

\section{OBSERVATIONS OF STRÖMGREN SPHERES IN} REDSHIFTED $21 \mathrm{~cm}$ EMISSION AND Ly $\alpha$ ABSORPTION

First, we consider the observed signatures of $\mathrm{H}$ II regions (Strömgren spheres) around the highest redshift quasars during their early relativistic expansion.

\subsection{Evolution of the Strömgren Sphere around a High-Redshift Quasar}

Neglecting recombinations that do not become important until the expansion becomes sub-relativistic, White et al. (2003) derived the evolution of the physical radius of the Strömgren Sphere $\left(R_{p}\right)$ as a function of the source age $\left(t_{\text {age }}\right)$, which can be easily extended to include the possibility of a nonzero radius $\left(R_{0}\right)$ at the initial time $t_{\text {age }}=0$,

$$
\dot{N}\left(t_{\text {age }}-\frac{R_{p}}{c}\right)=\frac{4 \pi}{3}\left(R_{p}^{3}-R_{0}^{3}\right) x_{\mathrm{H}} n_{\mathrm{H}}^{0}(1+z)^{3},
$$

where $\dot{N}$ is the production rate of ionizing photons by the quasar, $n_{\mathrm{H}}^{0}$ is the comoving hydrogen number density, $x_{\mathrm{H}}$ is 
the neutral fraction, and $c$ is the speed of light. This cubic equation may be solved algebraically (White et al. 2003). Alternatively, $R_{p}$ may be found by integrating the differential equation

$$
\frac{d R_{p}}{d t}=c\left[\frac{\dot{N}}{\dot{N}+4 \pi R_{p}^{2} c x_{\mathrm{H}} n_{\mathrm{H}}^{0}(1+z)^{3}}\right],
$$

with the initial condition $R_{p}(0)=R_{0}$. The latter approach (Wyithe \& Loeb 2004) allows for the inclusion of recombinations. As long as the number of photons emitted per time interval $\Delta t$ is larger than the number of hydrogens in a shell of thickness $\Delta R=c \Delta t$ at $R_{p}$, the $\mathrm{H}$ II region continues to expand at nearly the speed of light.

Calculations of $R_{p}$ in this paper assume that the ionization front is thin. The spectrum-averaged mean free path for the ionizing quasar photons is $\lambda \sim 1.5 x_{\mathrm{HI}}^{-1}(1+z)^{-3} \mathrm{Mpc}$, which may be compared to the bubble radius $R_{p} \sim 4.5 \mathrm{Mpc}$ (e.g., White et al. 2003), to yield the fractional thickness of the ionization front $f \equiv\left(\lambda / R_{p}\right) \sim 2 \times 10^{-3}\left(R_{p} / 4.5 \mathrm{Mpc}\right)^{-1} x_{\mathrm{H} \mathrm{I}}^{-1}[(1+z) / 7.3]^{-3}$. The assumption of a thin ionization front may be considered reasonable provided that $x_{\mathrm{H}} \gtrsim 10^{-2}$. A low neutral fraction is unlikely, given the size of the observed $\mathrm{H}$ II regions $\left(R_{p} \sim 4.5 \mathrm{Mpc}\right)$ around the $z \gtrsim 6.3$ quasars (Wyithe \& Loeb 2004). X-ray photons have a longer mean free path but are rare and do not contribute significantly to the number of ionizations.

\subsection{The Strömgren Sphere Radius Measured Through Ly $\alpha$ Absorption}

Equations (1) and (2) refer to the Strömgren sphere radius at a time $t_{\text {age }}$ after the source turns on. However, measurements of the radius through Ly $\alpha$ absorption $\left(R_{\mathrm{Ly}}\right)$ are made along the line of sight. Ly $\alpha$ photons emitted at $t_{\text {age }}$ are not absorbed until a time $t$ satisfying the relation $t=t_{\text {age }}+R(t) / c$ (note that the quasar may no longer be active at $t$ ). When considering the value of $R_{\mathrm{Ly}}$ measured through $\mathrm{Ly} \alpha$ absorption, the relevant time to consider is therefore the age of the quasar at the time when the photons being absorbed at the edge of the $\mathrm{H}$ II region were emitted $t_{\text {age, }}=t_{\text {age }}-R\left(t_{\text {age }}\right) / c$. In the absence of recombinations (White et al. 2003), the relation between $R_{\mathrm{Ly}}$ and $t_{\text {age, e }}$ is equivalent to the infinite speed-of-light solution for the evolution of a Strömgren sphere radius (Cen \& Haiman 2000; Madau \& Rees 2000),

$$
\begin{aligned}
R_{\mathrm{Ly}}= & {\left[R_{0}^{3}+\frac{3}{4 \pi} \frac{\dot{N}_{\|} t_{\mathrm{age}, \mathrm{e}}}{x_{\mathrm{H}} n_{\mathrm{H}}^{0}(1+z)^{3}}\right]^{1 / 3} } \\
= & 4 \mathrm{Mpc}\left[\left(\frac{R_{0}}{3.2 \mathrm{Mpc}}\right)^{3}\right. \\
& \left.+\left(\frac{\dot{N}_{\|}}{10^{57} \mathrm{~s}^{-1}}\right)\left(\frac{t_{\mathrm{age}, \mathrm{e}}}{10^{7} \mathrm{yr}}\right)\left(\frac{1+z}{7.5}\right)^{-3}\right]^{1 / 3}
\end{aligned}
$$

where we add the subscript $\|$ to denote the isotropic equivalent value of $\dot{N}$ for a measurement done parallel to the line of sight.

\subsection{The Strömgren Radius Measured through $\mathrm{H} \mathrm{I}$ Emission and Absorption}

Madau et al. (1997) and Tozzi et al. (2000) have considered the $\mathrm{H}$ I signature of the IGM surrounding a high-redshift quasar. Outside the $\mathrm{H}$ II region, neutral hydrogen may be heated by soft X-rays and by scattering of Ly $\alpha$ photons. The spin temperature of the neutral hydrogen is coupled to the kinetic temperature of the IGM through Ly $\alpha$ scattering of a background of UV photons and results in $21 \mathrm{~cm}$ emission (in excess of the CMB flux) around the quasar.

\subsubsection{The Lyo Scattering Rate}

The spin temperature of a warm IGM quickly becomes coupled to the CMB radiation, so that it yields no contrast for H I observations (Madau et al. 1997). However, scatterings of Ly $\alpha$ photons couple the spin temperature of the warm IGM at radii $R>R_{p}$ through the Wouthuysen-Field effect (Wouthuysen 1952; Field 1958). The spin temperature

$$
T_{s}=\frac{T_{\mathrm{CMB}}+y_{\alpha} T_{\mathrm{IGM}}}{1+y_{\alpha}}
$$

is weighted between the CMB temperature and the IGM temperature through the Ly $\alpha$ pumping efficiency

$$
y_{\alpha} \equiv \frac{4 P_{\alpha} T_{*}}{27 A_{10} T_{\mathrm{IGM}}} .
$$

In equation (4), $T_{*}=0.068 \mathrm{~K}$ is the temperature corresponding to the transition energy, $A_{10}=2.85 \times 10^{-15} \mathrm{~s}^{-1}$ is the spontaneous decay rate of the hyperfine transition, and $P_{\alpha}$ is the Ly $\alpha$ scattering rate per hydrogen.

The value of $P_{\alpha}$ may be calculated for a continuum UV source embedded in an expanding IGM around a Strömgren sphere as follows (see Loeb \& Rybicki 1999 for more details on the related physics). In the absence of scattering, photons that start at a frequency separation $\Delta \nu$ on the blue side of the Ly $\alpha$ resonance frequency $\nu_{\alpha}$ would redshift into resonance at some distance $R_{\text {res }}=\left(\Delta \nu / \nu_{\alpha}\right) c / H$ from the source, where $H=H(z)$ is the Hubble parameter at the quasar redshift. At this distance, the Hubble velocity of the IGM introduces a Doppler shift that is equal to the initial blueshift of these photons relative to the $\operatorname{Ly} \alpha$ line center. When a resonant photon enters the neutral IGM near $R_{\text {res }}$, its mean free path becomes very short because of the large cross section to Ly $\alpha$ scattering. As the photon frequency redshifts very close to the Ly $\alpha$ resonance, the mean free path diminishes, and the frequency shift per scattering (which corresponds to the Hubble velocity increment across a mean free path) goes to zero. Consequently, the photon spends a very long time around $R_{\text {res }}$. At this stage, the finite temperature of the gas becomes important. The photons are able to diffuse out of resonance primarily because of the Doppler shifts they encounter by the thermal speed of the atoms at $R_{\text {res }}$.

Each scattering near $R_{\text {res }}$ leads to a fractional frequency shift by the thermal Doppler effect of $\Delta \nu_{\text {th }} / \nu_{\alpha}=v_{\text {th }} / c$, where $v_{\text {th }}=\left(2 k T_{\mathrm{IGM}} / m_{p}\right)^{1 / 2}$ is the thermal speed of the atoms and $\nu_{\alpha}$ is the resonant Ly $\alpha$ frequency. The thermal shifts are of random sign, and so the photons go through a random walk in frequency. Consider the photons just around the resonant frequency at $R_{\text {res }}$. These photons change their frequency by $\sim \Delta \nu_{\text {th }}$ in each scattering and have a mean free path of $\lambda_{\text {th }}=1 /\left[n_{\mathrm{H}}^{0}(1+z)^{3} \sigma_{\text {th }}\right]$. Here $\sigma_{\text {th }}$ is the Ly $\alpha$ cross section at the center of the thermally broadened Ly $\alpha$ line (Rybicki \& Lightman 1979)

$$
\sigma_{\mathrm{th}}=\frac{\sqrt{\pi} e^{2}}{m_{e} c} \frac{f_{12}}{\Delta \nu_{\mathrm{th}}}=5.87 \times 10^{-12} \mathrm{~cm}^{2}\left(\frac{T_{\mathrm{IGM}}}{1 \mathrm{~K}}\right)^{-1 / 2}
$$


where $e$ and $m_{e}$ are the electron charge and mass, and $f_{21}$ is the oscillator strength for the Ly $\alpha$ transition. The mean intensity of nearly resonant photons at $R_{\text {res }}$ is increased by a factor of $\sim l / \lambda_{\text {th }}$ relative to optically thin conditions, where $l$ is the socalled Sobolev length, defined as the distance over which the bulk velocity gradient of the medium equals the thermal speed of an atom,

$$
l=\frac{v_{\mathrm{th}}}{d v / d R}=\frac{v_{\mathrm{th}}}{H},
$$

and $v=H R$ is the Hubble velocity. The optical-depth factor $\tau_{\text {th }}=l / \lambda_{\text {th }}$ reflects the fact that the diffusion speed of photons across the resonance region of width $\sim l$ is a factor $\lambda_{\text {th }} / l$ times smaller than the speed of light.

In a steady state, the mean intensity at all radii should therefore be enhanced relative to the optically thin case by a factor of $\sim \tau_{\text {th }}$. However, a steady state will not be established immediately when light from the quasar first penetrates the neutral IGM because of the slow diffusion speed of the resonant photons. At early times, scattering will produce an exponential reduction in resonant photon flux by a factor proportional to

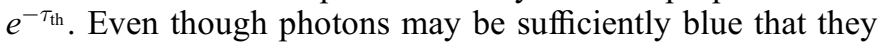
do not redshift into the resonance until $R_{\text {res }}$, they would still be absorbed by the blue wing of the Ly $\alpha$ resonance (where the cross section is still considerable) at radii smaller than $R_{\text {res }}$.

Let us therefore consider photons of arbitrary frequency on the blue side of the $\operatorname{Ly} \alpha$ resonance and calculate their mean intensity at $R_{\text {res }}$. We define the mean intensity as the angular average of the specific (directional) intensity $I(\nu, \mu)$, namely, $J(\nu)=\frac{1}{2} \int_{-1}^{1} d \mu I(\nu, \mu)$, where $\mu=\cos \theta$ is the direction relative to the radius vector at $R_{\text {res }}$ (Loeb \& Rybicki 1999). The Ly $\alpha$ optical depth for photons with frequency $\nu$ at a radius $R_{\text {res }}$ is

$\tau(\nu)=\int_{R_{\mathrm{res}}-\Delta R_{\mathrm{pl}}}^{R_{\mathrm{res}}} d R^{\prime} \sigma_{\alpha}\left\{\nu\left[1+\frac{H}{c}\left(R_{\mathrm{res}}-R^{\prime}\right)\right]\right\} n_{\mathrm{H}}^{0}(1+z)^{3}$,

where $\sigma_{\alpha}(\nu)=\sigma_{\text {th }} \exp \left[-\left(\nu-\nu_{\alpha}\right)^{2} / \Delta \nu_{\text {th }}^{2}\right]$ is the thermally broadened cross section (Rybicki \& Lightman 1979), and $\Delta R_{\mathrm{pl}}=R_{\mathrm{res}}-R_{p}(t-\Delta t)$ is the path length traversed by a photon through the neutral IGM after it had crossed the boundary of the $\mathrm{H}$ II region at a time $t-\Delta t$. In the strong scattering regime $[\tau(\nu) \gg 1]$, the mean intensity is a factor of $\tau(\nu)$ larger than the specific intensity along the radial direction, because the radial diffusion speed of the photons is $\sim c / \tau(\nu)$. The initial mean intensity of nearly resonant photons at radius $R_{\text {res }}$ is therefore

$$
J(\nu) \sim \frac{1}{4 \pi R_{\mathrm{res}}^{2}} \epsilon_{\text {local }}(\nu) \tau(\nu) e^{-\tau(\nu)},
$$

where

$$
\epsilon_{\text {local }}(\nu)=\epsilon\left[\nu\left(1+\frac{H R_{\text {res }}}{c}\right)\right]\left[\frac{H R_{\text {res }}}{c}+1\right]^{-1}
$$

is the redshifted source spectrum $\epsilon(\nu)$ in ergs s ${ }^{-1} \mathrm{~Hz}^{-1}$. The scattering rate $P_{\alpha}$ may then be calculated from the mean intensity as

$$
P_{\alpha}=\int_{0}^{\infty} d \nu \frac{J(\nu)}{h_{p} \nu} \sigma_{\alpha}(\nu)
$$

The directional intensity of resonant Ly $\alpha$ photons starts high near the boundary of the $\mathrm{H}$ II region at $R_{p}$ but is initially suppressed at larger radii. Resonant photons will diffuse to radii larger than $R_{p}$ at a speed $\sim c / \tau_{\text {th. }}$. In the case of a stationary $\mathrm{H}$ II front, the high-intensity region expands with time, extending a distance $\left(c / \tau_{\text {th }}\right) \Delta t$ beyond the edge of the $\mathrm{H}$ II region after a time $\Delta t$, and absorption will truncate the intensity at radii larger than $R_{p}+\left(c / \tau_{\text {th }}\right) \Delta t$. For the quasars under consideration, the expansion speed of the $\mathrm{H}$ II region $(z 0.1 c)$ is well in excess of the diffusion speed ${ }^{5}$ of resonant Ly $\alpha$ photons $\left(c / \tau_{\text {th }} \sim 10^{-5} c\right)$. On the timescales of interest here, there is a deficit of photons near the line center as described by equation (8).

The critical thermalization rate above which $\operatorname{Ly} \alpha$ scattering couples $T_{s}$ to $T_{\mathrm{IGM}}$ is given by

$$
P_{\mathrm{th}} \equiv \frac{27 A_{10} T_{\mathrm{CMB}}}{4 T_{*}} \sim 5 \times 10^{-12}\left(\frac{1+z}{7.5}\right) \mathrm{s}^{-1}
$$

(Madau et al. 1997). We find that $P_{\alpha}$ only exceeds the thermalization rate $P_{\text {th }}$ within a negligibly thin shell around the $\mathrm{H}$ II region $\left(\leqslant 10^{-4} \mathrm{Mpc}\right)$. As a result, detection of redshifted $21 \mathrm{~cm}$ emission from the $\mathrm{H}$ II regions of quasars will require the presence of a UV background. Background photons between the Ly $\alpha$ and the Lyman-limit frequencies can propagate through the universe and redshift with time to the point where they resonate with the $\mathrm{Ly} \alpha$ line and couple $T_{s}$ to $T_{\mathrm{K}}$. Such a background is expected to be present at $z \sim 6.5$, since the large electron scattering optical depth measured by WMAP (Kogut et al. 2003) implies that the IGM had been significantly reionized by that time (requiring the production of more than one ionizing photon per baryon above the Lyman limit).

Our result for the scattering rate should be compared with the result of Madau et al. (1997), who estimated

$$
\begin{aligned}
P_{\alpha}= & \int_{0}^{\infty} d \nu \frac{\epsilon(\nu)}{h_{p} \nu} \frac{1}{4 \pi R^{2}} \sigma_{\alpha}(\nu) \\
& \sim\left(8 \times 10^{-11} \mathrm{~s}^{-1}\right)\left(\frac{R}{1 \mathrm{Mpc}}\right)^{-2}\left[\frac{\nu_{\alpha} \epsilon\left(\nu_{\alpha}\right)}{3 \times 10^{46} \operatorname{ergs~s}^{-1} \mathrm{~Hz}^{-1}}\right] .
\end{aligned}
$$

In the steady state, this expression incorrectly neglects the rise in intensity that follows from the small diffusion speed of photons near the Ly $\alpha$ resonance and hence underestimates the value of $P_{\alpha}$ by a factor of $\sim n_{\mathrm{H}}^{0}(1+z)^{3} \sigma_{\text {th }} v_{\text {th }} / H$, which amounts to several orders of magnitude. However, in the initial state the expression neglects the absorption of resonant photons before they reach a radius $R>R_{p}$ and thus overestimates the value of $P_{\alpha}$ outside the $\mathrm{H}$ II region by several orders of magnitude.

The scattering of Ly $\alpha$ photons may also provide a source of heating for the gas. As this peak shifts to longer wavelengths, it loses energy to the hydrogen atoms off which the photons

\footnotetext{
${ }^{5}$ Note that the diffusion speed of a photon is a function of time as its frequency drifts in and out of resonance with the Ly $\alpha$ transition of the expanding IGM.
} 
scatter (Chen \& Miralda-Escude 2004). The additional energy density due to the scattering is

$$
U=\frac{\epsilon_{\text {local }}\left(\nu_{\alpha}\right)}{4 \pi R^{2} c}\left(\frac{l}{\lambda}-1\right) \Delta \nu_{\text {th }} \sim P_{\alpha} \frac{h_{p} \nu_{\alpha}}{c \sigma_{\text {th }}} .
$$

The rate at which energy is lost from the line in an expanding IGM equals the heating rate of the gas. Hence, we have

$$
\Gamma_{\alpha}=\frac{d U}{d t}=U \frac{1}{\nu} \frac{d \nu}{d t}=H P_{\alpha} \frac{h_{p} \nu_{\alpha}}{c \sigma_{\mathrm{th}}} .
$$

This heating rate, which is proportional to the $\operatorname{Ly} \alpha$ scattering rate, does not contribute to the heating of gas outside the $\mathrm{H}$ II region owing to the very low $\mathrm{Ly} \alpha$ intensity there. Instead, the heating is dominated by X-rays, which we discuss next.

\subsubsection{X-Ray Heating Beyond the $\mathrm{H}$ II Region}

Gas in the IGM outside the ionized region is subject to secondary electron heating resulting from ionizations by soft $\mathrm{X}$-rays that propagate into the neutral IGM beyond the $\mathrm{H}$ II region. The heating rate $\Gamma_{x}$ in ergs s $\mathrm{s}^{-1} \mathrm{Mpc}^{-3}$ at radius $R>R_{p}$ due to a source with a spectrum $\epsilon(\nu)$ (in ergs $\mathrm{s}^{-1} \mathrm{~Hz}^{-1}$ ) is

$$
\begin{aligned}
\Gamma_{x}(R, t)= & \int_{\nu_{\text {ion }}}^{\infty} d \nu f_{x} \frac{\epsilon_{\text {local }}(\nu)}{4 \pi R^{2}} \sigma_{\mathrm{pi}}(\nu) x_{\mathrm{H}} n_{\mathrm{H}}^{0}(1+z)^{3} \\
& \times \exp \left[-\Delta R_{\mathrm{pl}} \sigma_{\mathrm{pi}}(\nu) x_{\mathrm{H}} n_{\mathrm{H}}^{0}(1+z)^{3}\right],
\end{aligned}
$$

where $\sigma_{\mathrm{pi}}(\nu)$ is the cross section for photoionization and $\nu_{\text {ion }}=3.29 \times 10^{15} \mathrm{~Hz}$ is the Lyman-limit frequency corresponding to the ionization threshold of hydrogen. In equation (15), $\Delta R_{\mathrm{pl}}=R-R_{p}(t-\Delta t)$ is the path length of a photon through the neutral IGM after it crossed the boundary of the $\mathrm{H}$ II region at time $t-\Delta t$. We have neglected adiabatic cooling due to cosmological expansion in this expression, since the quasar lifetime is much shorter than the Hubble time. The fraction of photon energy converted into heat, $f_{x}$, may be evaluated using the fitting formula (Shull \& van Steenberg 1985)

$$
f_{x}=0.9971\left\{1-\left[1-\left(1-x_{\mathrm{HI}}\right)^{0.2663}\right]^{1.3163}\right\} \text {. }
$$

This equation is valid for photon energies $h_{p} \nu \gtrsim 100 \mathrm{eV}$. At lower energies, the value of $f_{x}$ may be roughly approximated by multiplying equation (16) with an additional factor of $\exp \left[\left(100 \mathrm{eV}-h_{p} \nu\right) / 100 \mathrm{eV}\right]$ (with the constraint that $f_{x}<1$ ). The residual ionization fraction from cosmological recombination of $\sim 5 \times 10^{-4}$ yields $f_{x} \sim 0.17$ (Madau et al. 1997), which sets the minimum heating rate. The heating rate leads to a temperature rise according to

$$
\frac{d T_{\mathrm{IGM}}}{d t}=\frac{2}{3} \frac{\Gamma_{x}}{k_{\mathrm{B}}} \frac{1}{x_{\mathrm{HI}} n_{\mathrm{H}}^{0}(1+z)^{3}} .
$$

\subsubsection{Redshifted $21 \mathrm{~cm}$ Observation of Quasar H II Regions}

The preceding discussion has shown that X-rays from the quasar are capable of heating the gas outside the $\mathrm{H}$ II region, and that the spin temperature of this warm gas can be coupled to its kinetic temperature in the presence of a strong UV ( $\operatorname{Ly} \alpha)$ background. In regions of the IGM in which the spin temperature is decoupled from the CMB temperature, a differential antenna temperature of

$$
\delta T_{b}=(23 \mathrm{mK}) x_{\mathrm{H} \mathrm{I}}\left(\frac{1+z}{7.5}\right)^{1 / 2}\left(\frac{T_{s}-T_{\mathrm{CMB}}}{T_{s}}\right)
$$

is observed. In equation (18) we have assumed a uniform IGM at the mean density with a neutral fraction $x_{\mathrm{H}}$. The luminous high-redshift quasars reside in rare high-density regions. However, on the scale of several megaparsecs, the overdensity due to infall around these regions is $\$ 10 \%$ (Barkana \& Loeb 2004a).

The brightness temperature of the redshifted $21 \mathrm{~cm}$ signal is small and subject to severe foreground contamination problems. Studies by Di Matteo et al. (2002) and Oh \& Mack (2003) have found that on scales of several arcminutes, randomly distributed point sources would have to be removed down to a level of $\sim 1 \mu \mathrm{Jy}$ before brightness temperature fluctuations on the order of $10 \mathrm{mK}$ could be detected. In the presence of the expected level of clustering of these sources, even the removal of point sources below $1 \mu \mathrm{Jy}$ would leave foreground fluctuations on this scale that are several orders of magnitude in excess of the redshifted $21 \mathrm{~cm}$ signal. However, the spectrum of the foreground contaminants is expected to be smooth (Shaver et al. 1999). The width of the $\mathrm{H}$ II region corresponds to a recessional velocity $v \lesssim 3000 \mathrm{~km} \mathrm{~s}^{-1}$ or a redshift interval of $\sim(1+z) v / c \sim 0.07$. The redshifted $21 \mathrm{~cm}$ ring will therefore produce a bump (or a dip) on top of the smooth foreground spectrum at the redshift of the quasar.

At a frequency $\nu_{\mathrm{bp}} \sim 120 \mathrm{MHz}$, an instrument like LOFAR is expected to have a bandpass of $\Delta \nu_{\mathrm{bp}} \sim 4 \mathrm{MHz}$. This should be compared with the frequency shift associated with the redshift difference between light emitted at the quasar and the edge of the $\mathrm{H}$ II region along the line of sight

$$
\Delta \nu \sim 2 \mathrm{MHz}\left(\frac{R_{\mathrm{Ly}}}{4 \mathrm{Mpc}}\right)\left(\frac{1+z}{7.5}\right) .
$$

Since many channels are contained within each bandpass, the structure of the $\mathrm{H}$ II region can therefore be probed along the line of sight in frequency space (Madau et al. 1997), allowing determination of the full three-dimensional shape of the $\mathrm{H}$ II region, including the radius of the $\mathrm{H}$ II region behind the quasar in addition to the radius $R_{\mathrm{Ly}}$ of the $\mathrm{H}$ II region in front of the quasar.

There are two distinct possibilities for the redshifted $21 \mathrm{~cm}$ signature of a high -redshift $\mathrm{H}$ II region. First, the region might be expanding into a cold IGM, in which case emission will only be seen out to a radius where the warming X-rays can reach. Alternatively, the IGM may have been preheated, either by an X-ray background, by gravitationally induced shocks in the IGM (Furlanetto \& Loeb 2003), or by an early reionization (e.g., Wyithe \& Loeb 2003a; Cen 2003). The presence of Gunn-Peterson troughs in quasar spectra at $z \gtrsim 6$ implies an IGM that is at least partially neutral, while the relatively small sizes of the Strömgren spheres around these quasars suggest a neutral fraction that is on the order of unity (Wyithe \& Loeb 2004). On the other hand, observations from WMAP (Kogut et al. 2003) suggest an optical depth to electron scattering of $\tau \sim 0.17$ and therefore significant reionization at $z \sim 20$. If 


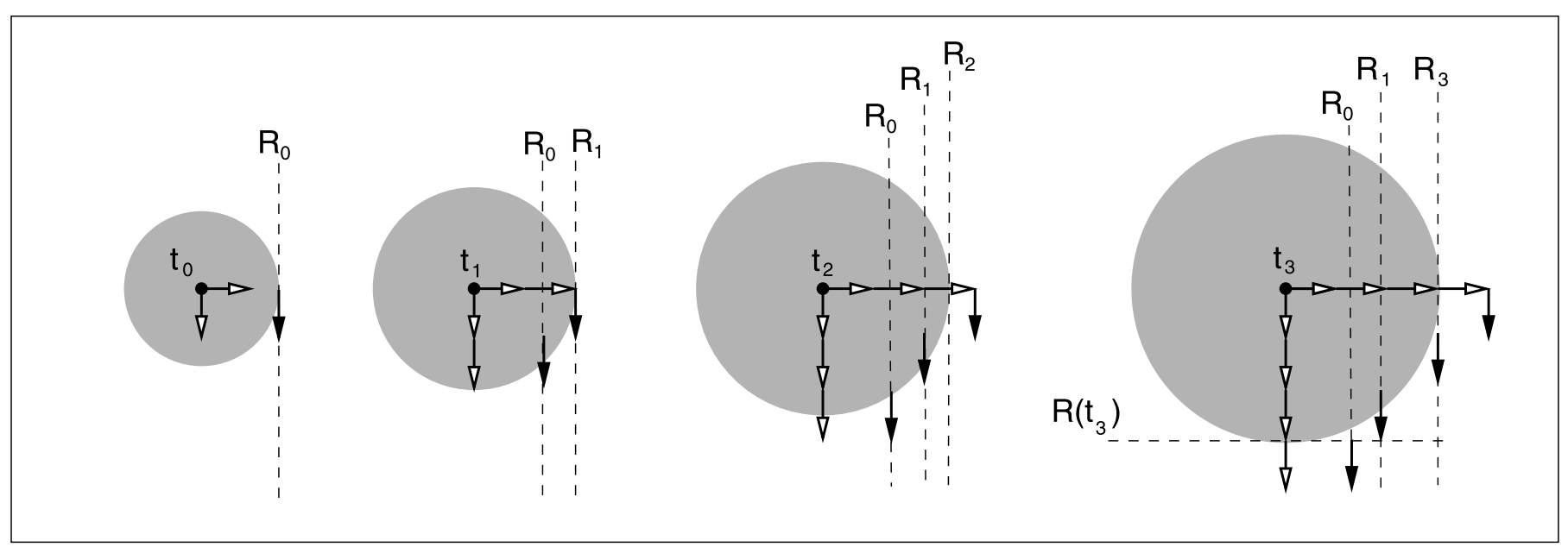

FIG. 1.-Schematic illustration of the geometry of Ly $\alpha$ and redshifted $21 \mathrm{~cm}$ observations of a relativistically expanding quasar Strömgren sphere (shaded regions). The four panels show the evolution at four different times. The quasar turns on at $t_{0}$, emitting photons isotropically into a preexisting $\mathrm{H}$ in region of radius $R_{0}$. Ly $\alpha$ photons (open arrows) emitted at $t_{0}$ reach the edge of the $\mathrm{H}$ II region at $t_{1}$. At that time, $21 \mathrm{~cm}$ photons (filled arrows) are emitted by the heated gas perpendicular to the line of sight (at the quasar redshift) at $R>R_{0}$ and travel toward the observer. At $t_{3}$, the Ly $\alpha$ photons emitted at $t_{1}$ reach the edge of the $\mathrm{H}$ II region. The absorption of these photons encodes a measurement of $R_{3}$. When these photons are observed, they are accompanied by $21 \mathrm{~cm}$ photons (emitted at $t_{1}$ from the quasars redshift), which show a ring of radius $R_{0}$.

the universe were reionized twice, then the Strömgren spheres would be expanding into a partially neutral, but warm IGM.

\section{COMPARISON OF STRÖMGREN SPHERE RADII MEASURED USING Ly $\alpha$ AND H I OBSERVATIONS}

The most natural place to search for the redshifted $21 \mathrm{~cm}$ signature of quasar $\mathrm{H}$ II regions is around the highest redshift quasars discovered in wide-field surveys such as the Sloan Digital Sky Survey (SDSS). ${ }^{6}$ Such quasars have H II regions that are also probed via their $\operatorname{Ly} \alpha$ absorption spectra. In this section we discuss the complimentary nature of the two observations with respect to the determination of the quasar lifetime, $t_{\mathrm{age}}$, and the neutral fraction of the IGM, $x_{\mathrm{HI}}$.

Strömgren spheres expand faster into a partially ionized IGM. If the IGM has a neutral fraction $x_{\mathrm{HI}}$, then the observation of $R_{\mathrm{Ly}}$ through Ly $\alpha$ absorption measures the combination $\left(t_{\text {age }, \mathrm{e}} / x_{\mathrm{HI}}\right)^{1 / 3}$ (where here we have neglected recombinations and assumed $R_{0}=0$ for simplicity). Detection of the redshifted $21 \mathrm{~cm}$ ring reflects the state of the $\mathrm{H}$ II region at $t_{\text {age, e. }}$. The differential antenna temperature $\delta T_{b}$ is proportional to $x_{\mathrm{H}}$ because of the small optical depth at $21 \mathrm{~cm}$,

$$
\delta T_{b} \propto\left(1-e^{-\tau_{\mathrm{H}}}\right) \approx \tau_{\mathrm{H} \mathrm{I}} \propto x_{\mathrm{H} \mathrm{I}} .
$$

Measurement of the contrast in the emission and absorption rings, or of the brightness temperature of the emission ring, will facilitate a measurement of $x_{\mathrm{H}}$ independent from $t_{\mathrm{age}, \mathrm{e}}$. Redshifted $21 \mathrm{~cm}$ observations may therefore provide an opportunity to break the degeneracy between $t_{\mathrm{age}, \mathrm{e}}$ and $x_{\mathrm{H}}$. Unfortunately, the heating rate and temperature of the IGM are uncertain, and unless $T_{k} \gg T_{\mathrm{CMB}}$, the value of $\delta T_{b}$ will be sensitive to the unknown value of $T_{k}$. Below we discuss an alternative geometric method for finding $x_{\mathrm{H}}$ that is not sensitive to the precise value of $\delta T_{b}$.

The relativistic expansion and finite light travel time imply that the $21 \mathrm{~cm}$ and Ly $\alpha$ measurements sample two different epochs in the evolution of the $\mathrm{H}$ II region. The situation is

\footnotetext{
${ }^{6}$ See http://www.sdss.org.
}

illustrated schematically in Figure 1. Suppose that the quasar turns on at $t_{0}$, emitting photons isotropically into an existing $\mathrm{H}$ II region of radius $R_{0}$. Ly $\alpha$ photons emitted at $t_{0}$ will reach the edge of the $\mathrm{H}$ II region at $t_{1}$. At that time, $21 \mathrm{~cm}$ photons with $\delta T_{b}>0$ are emitted by the heated gas at $R>R_{0}$ and travel toward the observer. For simplicity, consider $21 \mathrm{~cm}$ photons that are emitted from the heated IGM just beyond the ionizing front and perpendicular to the line of sight (i.e., at the quasar redshift). The sphere continues to expand at nearly the speed of light between $t_{1}$ and $t_{3}$. At $t_{3}$, the Ly $\alpha$ photons emitted at $t_{1}$ reach the edge of the $\mathrm{H}$ II region. The absorption of these photons encodes a measurement of $R_{3}$. The continuum and $21 \mathrm{~cm}$ photons propagate to the observer, who measures a radius $R_{3}$ along the line of sight in Ly $\alpha$ absorption, and a different radius $R_{0}$ in redshifted $21 \mathrm{~cm}$ photons.

In this paper we define $R_{\mathrm{Ly}}$ to be the radius measured through Ly $\alpha$ absorption along the line of sight. This is compared with $R_{\mathrm{H}}$, defined to be the radius of the ring measured perpendicular to the line of sight through redshifted $21 \mathrm{~cm}$ emission. The radii $R_{\mathrm{Ly}}$ and $R_{\mathrm{HI}}$ are not equal for a spherical region as a result of finite light travel time effects mentioned in the previous paragraph. We note that this effect is separate from the superluminal transverse expansion of the ring of $21 \mathrm{~cm}$ emission from the relativistically expanding portions of the $\mathrm{H}$ II region near the line of sight. These superluminally expanding rings would be seen $\sim 3000 \mathrm{~km} \mathrm{~s}^{-1}$ blueward of the quasar redshift (for $R_{p} \sim 4 \mathrm{Mpc}$ ).

The exact redshift of the quasar may be uncertain by up to $\sim 1000 \mathrm{~km} \mathrm{~s}^{-1}$ (Richards et al. 2002). However, this uncertainty corresponds to only $30 \%$ of the diameter of the $\mathrm{H}$ II region $\left(\sim 3000 \mathrm{~km} \mathrm{~s}^{-1}\right)$. A point on the edge of the $\mathrm{H}$ II region that is blueshifted by $\sim 1000 \mathrm{~km} \mathrm{~s}^{-1}$ relative to the quasar lies in a direction away from the quasar that makes an angle of $\beta \sim \cos ^{-1}\left(1000 \mathrm{~km} \mathrm{~s}^{-1} / 3000 \mathrm{~km} \mathrm{~s}^{-1}\right) \sim 70^{\circ}$ with the line of sight. As a result, the misidentification of the quasar redshift will lead to an error of $\sim R_{p}(1-\sin \beta) \sim 0.05 R_{p}$ in the transverse diameter of the ring at the quasar redshift. Moreover, since $\beta \gtrsim 70^{\circ}$, the ring of $21 \mathrm{~cm}$ emission at the observed quasar redshift will not be subjected to superluminal expansion, even if the quasar redshift is misidentified by $\sim 1000 \mathrm{~km} \mathrm{~s}^{-1}$. 

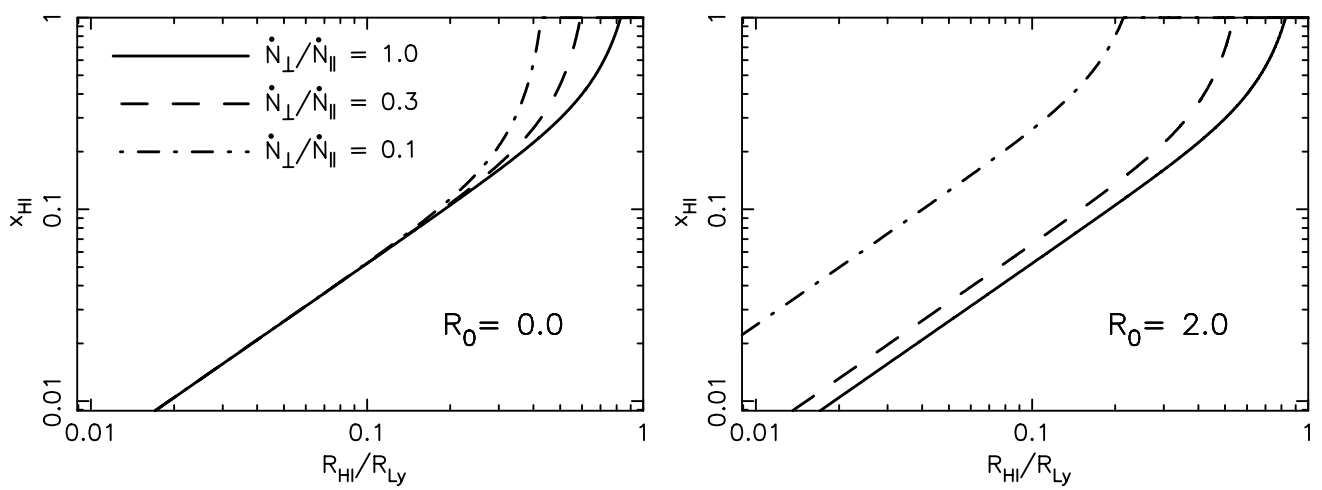

FIG. 2.-Neutral fraction as a function of the ratio $R_{\mathrm{HI}} / R_{\mathrm{Ly}}$ calculated from eq. (24). The calculations are for the case where $R_{\mathrm{Ly}}=4.5 \mathrm{Mpc}, z=6.5$, and $\dot{N}_{\|}=10^{57} \mathrm{~s}^{-1}$. We show curves for $\dot{N}_{\perp} / \dot{N}_{\|}=1.0,0.3$, and 0.1 , and for $R_{0}=0$ (left panel) and $R_{0}=2$ (right panel).

More quantitatively, the relativistic expansion and finite light travel time lead to several interesting possibilities:

1. The coupling of the spin temperature of neutral hydrogen to the temperature of the warm IGM surrounding the $\mathrm{H}$ II region results in redshifted $21 \mathrm{~cm}$ radiation in excess of the CMB. $R_{\mathrm{HI}}$ is measured transverse to the line of sight and thus corresponds to the radius at the time the observed UV photons were emitted. We can directly infer the ionizing photon rate $\left(\dot{N}_{\|}\right)$that drives the evolution of the $\mathrm{H}$ II region along the line of sight. However, since the quasar emission may not be isotropic, we allow the evolution of the $\mathrm{H}$ II region transverse to the line of sight to be driven by a different ionizing photon rate $\dot{N}_{\perp}$. Thus, the ratio $\dot{N}_{\perp} / \dot{N}_{\|}$is a measure of the anisotropy in quasar emission. Since high-redshift quasars are brightness selected, we assume that $\dot{N}_{\perp} / \dot{N}_{\|} \leq 1$. Using equation (3), we find the age of the quasar when light crossing the edge of the $\mathrm{H}$ II region was emitted $\left(t_{\text {age, }, \mathrm{e}}\right)$ and substitute it into equation (1) for the radius of the $\mathrm{H}$ II region at that time. This yields the following relationship between $R_{\mathrm{Ly}}$ and $R_{\mathrm{HI}}$ :

$$
\begin{aligned}
R_{\mathrm{Ly}}^{3}= & R_{0}^{3}\left(\frac{\dot{N}_{\|}}{\dot{N}_{\perp}}-1\right)+R_{\mathrm{H}}^{3}\left(\frac{\dot{N}_{\|}}{\dot{N}_{\perp}}\right) \\
& +\frac{R_{\mathrm{HI}}}{c} \frac{3}{4 \pi} \frac{\dot{N}_{\|}}{x_{\mathrm{H}} n_{\mathrm{H}}^{0}(1+z)^{3}},
\end{aligned}
$$

or

$$
\begin{aligned}
\left(\frac{R_{\mathrm{H} \mathrm{I}}}{R_{\mathrm{Ly}}}\right)^{3}= & \left(\frac{\dot{N}_{\perp}}{\dot{N}_{\|}}\right)-\left(\frac{R_{0}}{R_{\mathrm{Ly}}}\right)^{3}\left(1-\frac{\dot{N}_{\perp}}{\dot{N}_{\|}}\right) \\
& -\left[\left(\frac{R_{\mathrm{H} \mathrm{I}}}{R_{\mathrm{Ly}}}\right)\left(\frac{R_{\mathrm{Ly}}}{3.25 \mathrm{Mpc}}\right)^{-2}\right] \\
& \times\left[x_{\mathrm{H} \mathrm{I}}^{-1}\left(\frac{\dot{N}_{\perp}}{10^{57} \mathrm{~s}^{-1}}\right)\left(\frac{1+z}{7.5}\right)^{-3}\right] .
\end{aligned}
$$

Note that equations (21) and (22) are independent of $R_{0}$ when the quasar emission is isotropic, and that the dependence of $R_{\mathrm{HI}} / R_{\mathrm{Ly}}$ on $\dot{N}_{\perp} / \dot{N}_{\|}$is rather mild. If the quasar emission is isotropic, the transverse radius $R_{\mathrm{H}}$ is always smaller than the line-of-sight radius $R_{\mathrm{Ly}}$, particularly during the relativistic expansion phase. When the expansion becomes subrelativistic, $R_{\mathrm{HI}}$ approaches $R_{\mathrm{Ly}}$.
2. The radii measured through Ly $\alpha$ absorption and $21 \mathrm{~cm}$ emission correspond to different epochs separated by $R_{\mathrm{Ly}} / c$. For a spherical $\mathrm{H}$ II region we obtain the average expansion velocity of the ionizing front between the times corresponding to $R_{\mathrm{H} \mathrm{I}}$ and $R_{\mathrm{Ly}}$,

$$
\left\langle\frac{d R_{p}}{d t}\right\rangle=c\left(1-\frac{R_{\mathrm{HI}}}{R_{\mathrm{Ly}}}\right)
$$

Note that this velocity is the physical velocity of the ionizing front. The apparent transverse velocity of portions of the ring might be superluminal.

3. Given a fixed luminosity, the expansion speed at a radius $R_{\mathrm{Ly}}$ is proportional to $x_{\mathrm{HI}}^{-1 / 3}$, and so the neutral fraction can be inferred from the two radii. Equation (22) yields

$$
\begin{aligned}
x_{\mathrm{H} \mathrm{I}}= & {\left[\frac{R_{\mathrm{Ly}}}{c} \frac{\dot{N}_{\|}}{n_{\mathrm{H}}^{0}(1+z)^{3}}\right] } \\
& \times\left\{\frac{4 \pi}{3}\left[R_{\mathrm{Ly}}^{3}-R_{\mathrm{H}}^{3}\left(\frac{\dot{N}_{\|}}{\dot{N}_{\perp}}\right)-R_{0}^{3}\left(\frac{\dot{N}_{\|}}{\dot{N}_{\perp}-1}\right)\right]\right\}^{-1} \\
= & {\left[\frac{\left(R_{\mathrm{H}} / R_{\mathrm{Ly}}\right)}{1-\left(R_{\mathrm{H}} / R_{\mathrm{Ly}}\right)^{3}\left(\dot{N}_{\|} / \dot{N}_{\perp}\right)-\left(R_{0} / R_{\mathrm{Ly}}\right)^{3}\left[\left(\dot{N}_{\|} / \dot{N}_{\perp}\right)-1\right]}\right] } \\
& \times\left(\frac{R_{\mathrm{Ly}}}{3.25 \mathrm{Mpc}}\right)^{-2}\left(\frac{\dot{N}_{\|}}{10^{57} \mathrm{~s}^{-1}}\right)\left(\frac{1+z}{7.5}\right)^{-3} .
\end{aligned}
$$

Some examples of the dependence of $x_{\mathrm{HI}}$ on the ratio $\left(R_{\mathrm{H}} / R_{\mathrm{Ly}}\right)$ given $R_{\mathrm{Ly}}=4.5 \mathrm{Mpc}$ and $\dot{N}_{\|}=10^{57} \mathrm{~s}^{-1}$ are shown in Figure 2. In the case of isotropic quasar emission $\left(\dot{N}_{\perp} / \dot{N}_{\|}=\right.$ $1)$, a small ratio of $\left(R_{\mathrm{HI}} / R_{\mathrm{Ly}}\right)$ implies relativistic expansion and hence a small neutral fraction, unless $R_{\mathrm{Ly}} \ll 3.25 \mathrm{Mpc}$. Values of $\dot{N}_{\perp} / \dot{N}_{\|}$that are less than unity lead to larger inferred values of $x_{\mathrm{H}}$. Similarly, a nonzero value for $R_{0}$ leads to larger values of $x_{\mathrm{HI}}$. The assumptions of isotropy and $R_{0}=0$ lead to determination of a lower limit for $x_{\mathrm{H}}$. The observation of a redshifted $21 \mathrm{~cm}$ ring with a radius greater than $2.5 \mathrm{Mpc}(0.5 \mathrm{Mpc})$ around the known $z \gtrsim 6.3$ quasars (with $R_{\mathrm{Ly}} \sim 4.5 \mathrm{Mpc}$ ) would provide a limit on the neutral fraction of $x_{\mathrm{H} \mathrm{I}}>0.3\left(x_{\mathrm{HI}}>0.05\right)$.

\section{SOME EXAMPLES}

In this section we present examples of the redshifted $21 \mathrm{~cm}$ signatures of $z>6$ quasars that will be observed by instruments 


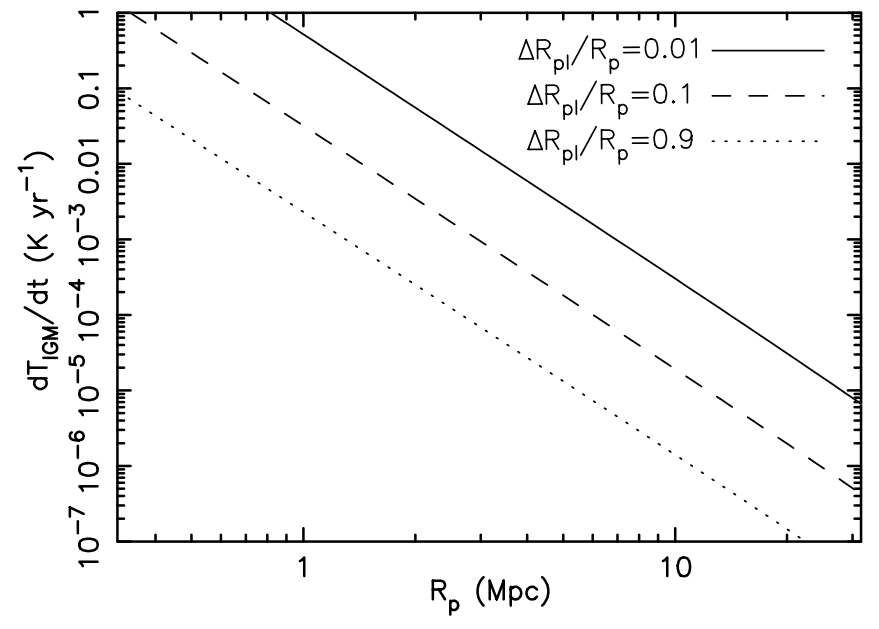

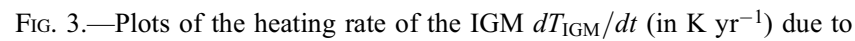
the X-ray emission by the quasar, as a function of the Strömgren radius. The three curves correspond to radii at which the photons have traversed $\mathrm{H}$ I path lengths of $\Delta R_{\mathrm{pl}}=0.01 R_{p}, 0.1 R_{p}$, and $0.9 R_{p}$, where $R_{p}$ is the physical radius of the $\mathrm{H}$ II region. The assumed spectrum is given in eq. (25), and the neutral fraction $x_{\mathrm{H}}$ was set to unity.

such as LOFAR. The UV continuum blueward of Ly $\alpha$ is assumed to be that of the median spectrum in the sample of Hubble Space Telescope quasars studied by Telfer et al. (2002). They found a power law $\epsilon_{c}(\nu) \propto \nu^{-\alpha_{\mathrm{EUV}}}$ with $\alpha_{\mathrm{EUV}}=1.57$. This slope may be extended into the X-ray regime based on the results of Yuan et al. (1998). We include the Ly $\alpha$ emission line centered at $\lambda_{\alpha}=912 \AA$, with an equivalent width of $W_{\lambda}=90 \AA$ (Vanden Berk et al. 2001; Telfer et al. 2002) and a line width of $\sigma_{\lambda}=19 \AA$ (Vanden Berk et al. 2001), providing a UV-X-ray spectrum

$$
\begin{aligned}
\epsilon(\nu)= & 1.3 \times 10^{31} \operatorname{ergs~s}^{-1} \mathrm{~Hz}^{-1}\left(\frac{\nu}{\nu_{\text {ion }}}\right)^{-\alpha_{\mathrm{EUV}}} \\
& \times\left\{1+\frac{1}{\sqrt{2 \pi} \sigma_{\lambda}} \exp \left[\frac{\left(c / \nu-\lambda_{\alpha}\right)^{2}}{\sigma_{\lambda}^{2}}\right]\right\} .
\end{aligned}
$$

The normalization of this spectrum is appropriate for a $2 \times$ $10^{9} M_{\odot}$ supermassive black hole accreting at its Eddington rate (Elvis et al. 1994), and it yields an ionizing photon rate of $\dot{N}_{\|}=1.3 \times 10^{57} \mathrm{~s}^{-1}$, representative of the $z \gtrsim 6.3$ SDSS quasars (White et al. 2003). In this section, we show examples of the $21 \mathrm{~cm}$ emission signal that may be observed around the known very high redshift quasars, with the spectrum described by equation (25).

The level of redshifted $21 \mathrm{~cm}$ emission is enhanced through heating of the IGM by the quasar X-ray emission. The corresponding heating rates $d T_{\mathrm{IGM}} / d t$ of the above quasar spectrum are plotted as a function of radius in Figure 3. The three curves correspond to radii where photons traversed path lengths through neutral gas of $\Delta R_{\mathrm{pl}}=0.01 R_{p}, 0.1 R_{p}$, and $0.9 R_{p}$. The neutral fraction was set to unity in these examples. Note that lower neutral fractions lead to higher values of $f_{x}$ and hence larger heating rates.

If the Strömgren spheres expand into a preheated IGM containing a UV background that coupled the spin temperature of the IGM to its kinetic temperature, the $\mathrm{H}$ II regions should appear as holes in the sky of redshifted $21 \mathrm{~cm}$ emission. As an example, we first consider an IGM that is pre- heated to $\sim 100 \mathrm{~K}$ at $z=6.3\left(T_{\mathrm{IGM}} \sim 5 T_{\mathrm{CMB}}\right)$. We follow the evolution of the Strömgren sphere and at each time compute the heating rate of the IGM beyond the edge of the $\mathrm{H}$ II region using equation (15). The evolution of the kinetic temperature of the IGM is followed on a grid using equation (17). The spin temperature is then set to $T_{\text {IGM }}$ as appropriate in the presence of a strong Ly $\alpha$ background. Figure 4 shows four examples of the $21 \mathrm{~cm}$ emission signature of a Stromgren sphere expanding into a warm IGM as a function of radius (top axes) and apparent angle (bottom axes) for different combinations of $\dot{N}_{\perp} / \dot{N}_{\|}=1.0,0.1$ and $x_{\mathrm{H} \text { I }}=1.0,0.1$. An initial value of $R_{0}=0$ was assumed. We have restricted ours attention to $x_{\mathrm{H}} \geq 0.1$ since the next generation instruments will not be able to detect the brightness temperature contrast at lower neutral fractions. This regime of $x_{\mathrm{H}} \geq 0.1$ is suggested by analysis of the size of the observed $\mathrm{H}$ II regions around the highest redshift quasars (Wyithe \& Loeb 2004), but we note that lower neutral fractions may render the edge of the $\mathrm{H}$ II region unobservable in redshifted $21 \mathrm{~cm}$ emission. The $y$-axes are labeled by the differential antenna temperature $\delta T_{b}$ (right axes), and the flux $\delta I$ (left axes) assuming the design goal sensitivity of LOFAR

$$
\delta I=\left(1.2 \mu \mathrm{Jy} \operatorname{arcmin}^{-2}\right)\left(\frac{\delta T_{b}}{10 \mathrm{mK}}\right)\left(\frac{1+z}{7.5}\right)^{-2}
$$

for a top-hat beam with a diameter of $2^{\prime}$. In each case, four profiles are shown for observing times corresponding to quasar ages of $t_{\mathrm{age}, \mathrm{e}}=0.5,1,2,4 \times 10^{7} \mathrm{yr}$ at the time when the optical photons observed along the line of sight were emitted. The lower row includes cases with $x_{\mathrm{H}}=0.1$. The smaller neutral fraction results in larger radii, faster relativistic expansion, and therefore thinner emission shells. The $21 \mathrm{~cm}$ emission signal is weaker in this case because $\delta T_{b} \propto x_{\mathrm{HI}}$. There is excess emission above the level of the general IGM in a ring covering the region where gas has been heated by the quasar. However, the brightness temperature is only slightly higher than the rest of the IGM. This is due to the temperature dependence of $\Delta T_{b}$, which asymptotes to a constant value of $\sim 23 x_{\mathrm{H}} \mathrm{mK}$ for high temperatures. The panels on the right show examples with $\dot{N}_{\perp} / \dot{N}_{\|}=0.1$. The lower transverse flux results in an expansion that becomes subrelativistic at earlier times. As a result, the emission shell has a smaller radius and a larger thickness.

As our second example, we consider an IGM that is cold in neutral regions $\left[T \sim 0.026(1+z)^{2}\right.$, corresponding to adiabatic cooling following the thermal decoupling of the baryons from the CMB]. This situation may arise if the massive quasars form before there has been sufficient time for an X-ray background to heat the IGM. The resulting $21 \mathrm{~cm}$ signal is shown in Figure 5. The heated portions of the IGM beyond the edge of the $\mathrm{H}$ II region show emission, while the cold IGM produces absorption of the $\mathrm{CMB}$ at larger radii. The brightness temperature of the emission ring obtains values of $T_{b} \sim 20 x_{\mathrm{H}}$ $\mathrm{mK}$, smaller in amplitude than the absorption signal of $T_{b} \sim-295 x_{\mathrm{H}} \mathrm{mK}$. A cold IGM therefore offers the best contrast for observation of the transverse extent of the $\mathrm{H}$ II region. As in the previous example, the emission rings get thicker at late times because the expansion speed becomes less relativistic. Since the transverse expansion becomes subrelativistic sooner than the line-of-sight expansion, the rings become thick at earlier times for a lower value of $\dot{N}_{\perp} / \dot{N}_{\|}$. 

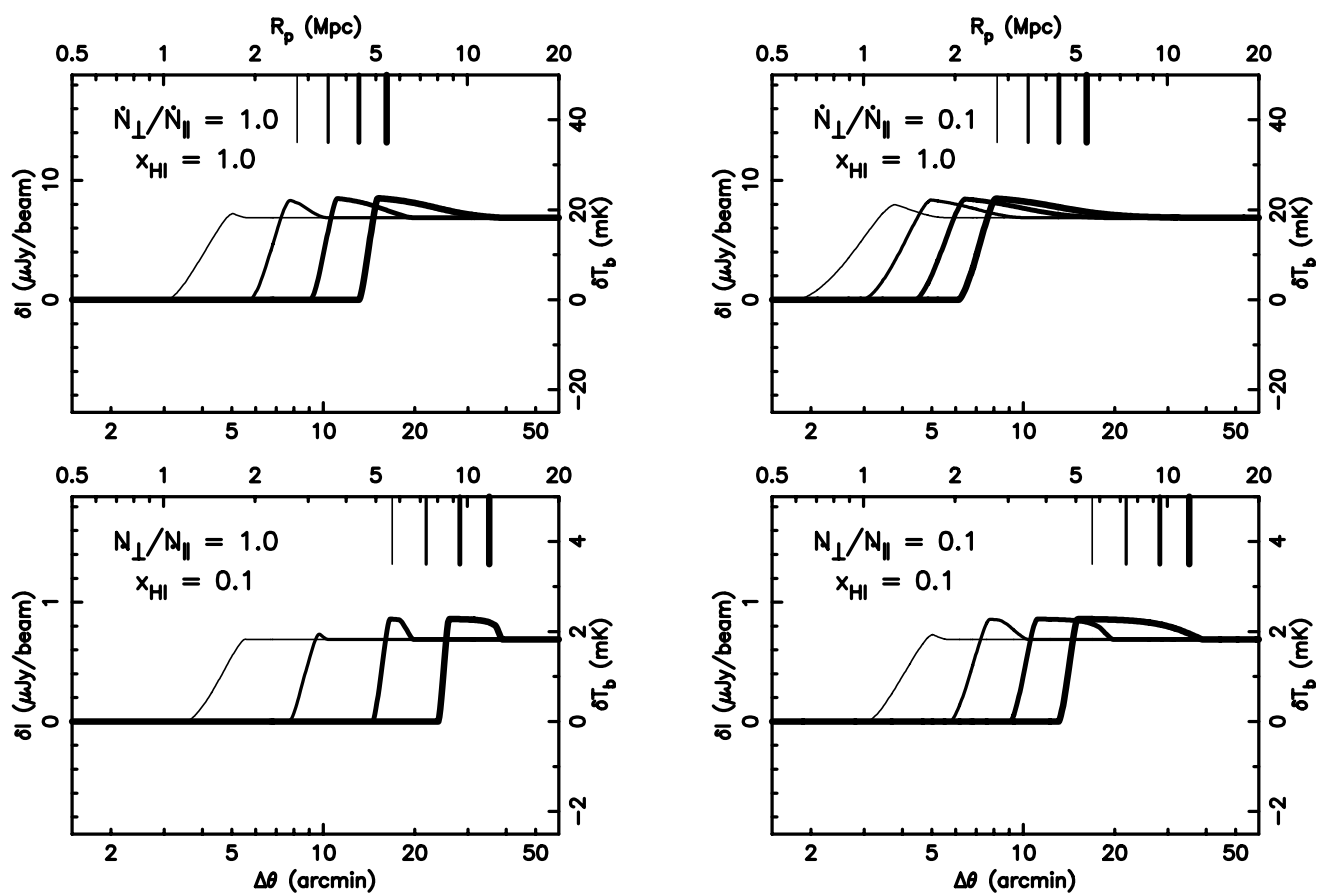

FIG. 4.-Four examples of the evolution of a Strömgren sphere expanding into a warm $\left(T_{\mathrm{IGM}}=5 T_{\mathrm{CMB}}\right)$ IGM as seen in redshifted $21 \mathrm{~cm}$ emission and Ly $\alpha$ absorption, assuming different combinations of $\dot{N}_{\perp} / \dot{N}_{\|}=1.0,0.1$ and $x_{\mathrm{H}}=1.0,0.1$. In all cases we assume $R_{0}=0$. The $21 \mathrm{~cm}$ emission signal is plotted as a function of radius (upper horizontal axes) and apparent angle in arcminutes (lower axes). The $y$-axes are labeled by the differential antenna temperature $\delta T_{b}$ (right axes) and the flux $\delta I$ (left axes) given a circular top-hat beam with a diameter of $2^{\prime}$. In each case, four profiles are shown for observing times corresponding to quasar ages of $t_{\mathrm{age}, \mathrm{e}}=0.5,1,2,4 \times 10^{7} \mathrm{yr}$ when the optical photons were emitted. Values of $R_{\mathrm{Ly}}$ at $t_{\mathrm{age}, \mathrm{e}}=0.5,1,2,4 \times 10^{7} \mathrm{yr}$ are shown with tick marks.

Figure 6 shows $R_{\mathrm{Ly}}\left(t_{\mathrm{age}}\right)$ (solid dark lines) as well as the inner radius of the $21 \mathrm{~cm}$ emission ring (solid light lines) for the example presented in the top left panels of Figures 4 and 5 $\left(\dot{N}_{\perp} / \dot{N}_{\|}=1, x_{\mathrm{H} \text { I }}=1\right)$. The four epochs corresponding to the differential antenna temperature profiles plotted in Figures 4 and 5 are indicated by the vertical tick marks. Similarly, values of $R_{\mathrm{Ly}}$ at $t_{\mathrm{age}, \mathrm{e}}=0.5,1,2,4 \times 10^{7} \mathrm{yr}$ are indicated by tick marks in the panels of Figures 4 and 5. The value of $R_{\mathrm{Ly}}$ is always larger than the radius of $21 \mathrm{~cm}$ emission. The dashed lines show the velocity at which the edge of the $\mathrm{H}$ II region is moving when observed at a time corresponding to $t_{\mathrm{age}, \mathrm{e}}$. The $21 \mathrm{~cm}$ ring (light dashed lines) is observed at an earlier
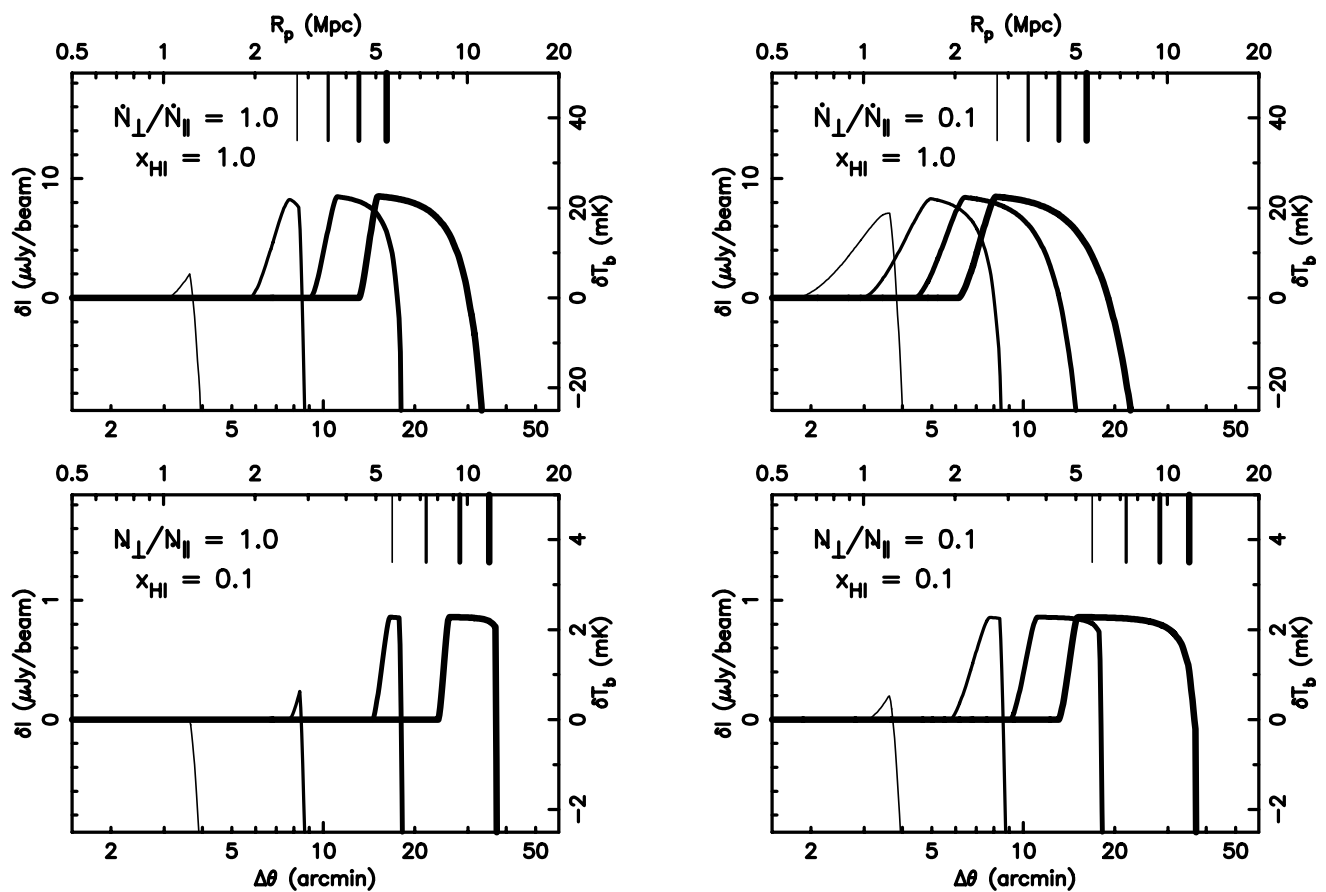

FIG. 5.-Same as Fig. 4, but with a cold IGM having $T_{\mathrm{IGM}}=0.026(1+z)^{2} \ll T_{\mathrm{CMB}}$. 


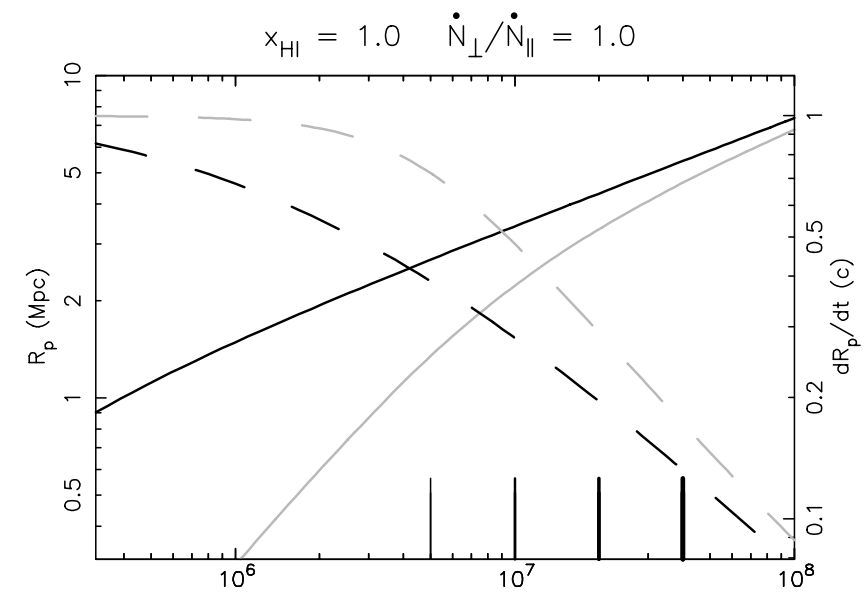

FIG. 6.-Plots of the radius measured through Ly $\alpha$ absorption along the line of sight $R_{\mathrm{Ly}}\left(t_{\mathrm{age}}\right)$ (dark solid lines) as well as the radius of the interior boundary of the $21 \mathrm{~cm}$ emission ring at the quasar redshift (light solid lines). The dashed lines show the speed at which the edge of the $\mathrm{H}$ II region seen in $21 \mathrm{~cm}$ (light dashed lines) and Ly $\alpha$ absorption (dark dashed lines) is moving when observed at a time corresponding to $t_{\text {age, }}$. The curves correspond to the example shown in the top left panel of Fig. 4 with $\dot{N}_{\perp} / \dot{N}_{\|}=1$ and $x_{\mathrm{H}}=1$.

phase of evolution and thus is moving at a higher velocity. The edge of the $\mathrm{H}$ II region is moving at a speed $\gtrsim 0.2 c$ when $R_{p} \lesssim 4.5 \mathrm{Mpc}$.

\section{FOSSIL H II REGIONS IN THE HIGH REDSHIFT IGM}

The presence of a Ly $\alpha$ background has very interesting implications for the observation of fossil $\mathrm{H}$ II regions. The recombination time within an ionized region of the IGM at mean density is $t_{\text {recom }} \sim\left[\alpha_{B} n_{\mathrm{H}}^{0}(1+z)^{3}\right]^{-1} \sim 1.5 \times 10^{9}[(1+z) / 7.5]^{-3} \mathrm{yr}$, where $\alpha_{B}=2.6 \times 10^{-13} \mathrm{~cm}^{3} \mathrm{~s}^{-1}$ is the case $\mathrm{B}$ recombination coefficient at a temperature of $10^{4} \mathrm{~K}$. Thus, the recombination time at the mean cosmic density exceeds the Hubble time for $z \lesssim 7$. Once a quasar has turned off, it leaves behind a fossil $\mathrm{H}$ II region that survives recombination. This $\mathrm{H}$ II region will therefore remain detectable in redshifted $21 \mathrm{~cm}$ radiation in both scenarios discussed in this paper. Since the bright SDSS quasars are likely to be active for only $\sim 1 \%$ of the time (Wyithe \& Loeb 2003b), there should be on the order of 100 times as many $21 \mathrm{~cm}$ emission rings around fossil $\mathrm{H}$ II regions as there are around active quasars. Furthermore, the hierarchical buildup of the supermassive black holes powering the quasars would result in an even larger number of small fossil $\mathrm{H}$ II regions. At redshifts greater than 7 , the $\mathrm{H}$ II region may still persist as a result of the stellar contribution to the ionizing radiation. Fossil H II regions containing significant stellar luminosity may also exhibit a Ly $\alpha$ halo of the type discussed by Loeb \& Rybicki (1999). The detection of such a Ly $\alpha$ halo can be used to calibrate the stellar ionizing background.

How many $\mathrm{H}$ II regions should an instrument like LOFAR detect? It is anticipated that near frequencies of $\nu_{\mathrm{bp}} \sim$ $200 \mathrm{MHz}$, the virtual core of the planned instrument LOFAR will have a beam size of $\Delta \theta_{\text {beam }} \sim 23^{\circ}$, a resolution of several tens of arcminutes, and a system temperature of $T_{\text {sys }} \sim 300 \mathrm{~K}$. The instrument will have an effective collecting area of $A \sim$ $10^{5} \mathrm{~m}^{2}$ at this frequency, leading to a resolution of $\Delta \theta_{\mathrm{fa}} \sim 1.22 \lambda$ $(\pi / A)^{1 / 2} \sim 22^{\prime}$ for the telescope in a filled aperture mode. Higher resolutions are obtained by spreading the array out over longer baselines, at the cost of decreased sensitivity. From the radiometer equation (e.g., Burke \& Graham-Smith
1997) we can find the rms noise in the brightness temperature as a function of resolution on angular scales $\Delta \theta<\Delta \theta_{\text {fa }}$ of

$$
\begin{aligned}
\Delta T_{b}(\Delta \theta)= & (0.24 \mathrm{mK})\left(\frac{\Delta \theta}{\Delta \theta_{\mathrm{res}}}\right)^{2}\left(\frac{T_{\mathrm{sys}}}{300 \mathrm{~K}}\right) \\
& \times\left(\frac{\Delta \nu_{\mathrm{bp}}}{4 \mathrm{MHz}}\right)^{-1}\left(\frac{\tau}{100 \mathrm{hr}}\right)^{-1 / 2}
\end{aligned}
$$

where $\Delta \nu_{\mathrm{bp}}$ is the detector bandpass, and $\tau$ is the integration time. The noise in the brightness temperature is constant on scales larger than $\Delta \theta_{\mathrm{fa}}$. The rms noise for an observation with a resolution $\Delta \theta \sim 3^{\prime}$ therefore becomes comparable to the expected emission signal $\left(\delta T_{b} \sim 10 \mathrm{mK}\right)$ for a modest integration time of $\tau \sim 100 \mathrm{hr}$.

The comoving optical luminosity function of bright quasars at $z \sim 6$ in units of $L_{\odot}^{-1} \mathrm{Gpc}^{-3}$ (Fan et al. 2003) is

$$
\Phi(L) \approx 10^{-13} L_{\odot}^{-1} \mathrm{Gpc}^{-3}\left(\frac{L}{10^{13.1} L_{\odot}}\right)^{\beta}
$$

where $\beta \sim-3$ is the logarithmic slope at the bright end. Given a bandpass $\Delta \nu_{\mathrm{bp}} \sim 4 \mathrm{MHz}$ and a central frequency $\nu_{\mathrm{bp}} \sim$ $200 \mathrm{MHz}$, the number of active quasars per logarithm of luminosity per field of view is

$$
N \sim L \Phi(L) \frac{d^{2} V}{d z d \Omega} \pi\left(\frac{\Delta \theta_{\mathrm{beam}}}{2}\right)^{2}(1+z) \frac{\Delta \nu_{\mathrm{bp}}}{\nu_{\mathrm{bp}}},
$$

where $d^{2} V / d z d \Omega$ is the comoving volume per unit redshift per unit solid angle. Equation (29) yields a value of $N \sim 2$ bright $z \sim 6$ quasars per LOFAR field.

The number of redshifted $21 \mathrm{~cm}$ rings or holes in the emission signature of the IGM per field as determined by equation (29) applies to active quasars. However, as noted above, the $21 \mathrm{~cm}$ emission may persist after the quasar has turned off. This is because in the absence of other heating sources, gas heated to temperatures far in excess of the CMB will cool adiabatically. The $(1+z)^{2}$ dependence of the temperature in this regime should ensure that the gas remains at a temperature in excess of the CMB by the time it is reheated during the next episode of quasar activity. We might expect to find significant numbers of fossil $\mathrm{H}$ II regions, provided that there is continued coupling of $T_{s}$ and $T_{\mathrm{IGM}}$ due to a UV background. The number of detectable fossil $\mathrm{H}$ II regions would then equal the number in equation (29) multiplied by the inverse of the quasar duty cycle $\sim 1 \%$ (Wyithe \& Loeb 2003 b), or about 200 per field.

LOFAR should also be sensitive to $\mathrm{H}$ II regions around less luminous quasars. The brightness temperature of the emission ring, or of the IGM outside the $\mathrm{H}$ II region, has a mild dependence on the quasar luminosity, and its diameter scales only with the one-third power of the luminosity. $\mathrm{H}$ II regions around less luminous quasars should be more numerous by a factor proportional to $L^{\beta+1}$. For example, detection of redshifted $21 \mathrm{~cm}$ emission around quasars with luminosities a factor of 10 smaller than those of the bright SDSS quasars require a resolution that is better by a factor of $10^{1 / 3}$, corresponding to an integration time that is longer by a factor of $10^{2 / 3} \sim 4.6$. However, the number of active high-redshift quasars per field powering such emission rings is likely to be 
on the order of $10^{2} N \sim 200$, while the number of fossils regions could be $\sim 2 \times 10^{4}$.

Finally, we briefly discuss the example of a redshifted $21 \mathrm{~cm}$ signature around a high-redshift quasar presented in Figure $5 b$ of Tozzi et al. (2000). Their example shows a ring of redshifted $21 \mathrm{~cm}$ emission inside a ring of redshifted $21 \mathrm{~cm}$ absorption. This morphology is unlikely to be observed because the observation of an absorption ring requires the absence of a UV background, and the provision of coupling of the spin and kinetic temperatures through $\operatorname{Ly} \alpha$ photons from the quasar itself. As we have shown, Ly $\alpha$ photons from the quasar are unable to couple the spin and kinetic temperatures. An emission ring enclosed within an IGM that absorbs everywhere, as shown in Figure 5 is the correct signature of a Strömgren sphere expanding into a cold IGM (provided that there is a UV background).

\section{STRÖMGREN SPHERES AND THE GLOBAL SIGNATURE OF REIONIZATION}

A very exciting possibility for instruments like LOFAR is to search for the emission (or absorption) of redshifted $21 \mathrm{~cm}$ radiation from the neutral IGM before reionization. By finding the frequency at which this emission (absorption) cuts off, one might determine the redshift of reionization (e.g., Shaver et al. 1999). If reionization took place over an extended period of time, as suggested by recent work (Barkana \& Loeb 2004b), then the global reionization signature will be difficult to detect for two primary reasons: (1) because it will be superposed on a smooth but varying foreground spectrum and (2) because calibration needs to be performed over a frequency range that is broader than the detector bandpass.

Arcminute resolution observations of quasar Strömgren spheres would provide an alternative method to search for a step in the brightness temperature of the IGM due to reionization. This is because the inner part of the $\mathrm{H}$ II region provides a calibration of the brightness temperature of the radio foreground that is unavailable along other lines of sight. Note that $21 \mathrm{~cm}$ emission from the quasar itself does not affect this calibration, since it effectively just adds to the foreground. Observations along the line of sight to the quasar would include emission within the spectral ranges corresponding to both the ionized region and the neutral gas within the same bandpass. Reshifted $21 \mathrm{~cm}$ observations of quasars at a series of redshifts would therefore allow determination of the brightness temperature of the IGM at different cosmic times, and a detection of the global signature of reionization. Redshifted $21 \mathrm{~cm}$ observation of highest redshift SDSS quasars will immediately determine whether the IGM was significantly neutral at $z>6.3$.

\section{DISCUSSION}

Observations of the reionization epoch in redshifted $21 \mathrm{~cm}$ radiation with proposed instruments such as LOFAR will offer a unique opportunity to study the state of the IGM at the end of the dark ages. The warm neutral hydrogen outside the $\mathrm{H}$ II regions surrounding bright high-redshift quasars is expected to emit at $21 \mathrm{~cm}$. The brightness temperature contrast of this emission will probe the neutral fraction (with a lower contrast implying a lower neutral fraction), although the inference will be subject to uncertainties in the temperature of the warm IGM (unless the IGM temperature is much greater than that of the $\mathrm{CMB}$ ). Alternatively, the relativistic expansion of the $\mathrm{H}$ II regions provides a geometric method to determine the neutral fraction as well as the quasar lifetime.

We have combined the observed $\operatorname{Ly} \alpha$ absorption signal with expectations for redshifted $21 \mathrm{~cm}$ signatures of the $\mathrm{H}$ II regions around the highest redshift SDSS quasars. Because the $\mathrm{H}$ II regions around the known $z \gtrsim 6.3$ quasars are at a stage of relativistic expansion, the line-of-sight and transverse radii (measured through Ly $\alpha$ absorption and redshifted $21 \mathrm{~cm}$ emission, respectively) correspond to two different epochs in the evolution of the $\mathrm{H}$ II regions. We have found that measurements of these two radii can be used to determine the neutral fraction of the IGM with only a mild dependence on the anisotropy of the quasar emission (see Fig. 2). Assuming isotropic quasar emission and the absence of a fossil $\mathrm{H}$ II region prior to the quasar activity, the observation of a redshifted $21 \mathrm{~cm}$ emission ring with a radius greater than $2.5 \mathrm{Mpc}$ $(0.5 \mathrm{Mpc})$ around the known $z \gtrsim 6.3$ quasars would provide a lower limit on the neutral fraction of $x_{\mathrm{H}}>0.3\left(x_{\mathrm{H}}>0.05\right)$. Emission concentrated close to the line of sight and the existence of fossil H II regions around the quasar host galaxy would imply even larger neutral fractions.

At low redshifts, quasar lifetimes are bracketed by a variety of techniques to the range $\sim 10^{6}-10^{8} \mathrm{yr}$ (e.g., Yu \& Tremaine 2002; Martini \& Weinberg 2001; Haiman \& Hui 2001; Jakobsen et al. 2003), with preferred values near $10^{7}$ yr (e.g., Martini 2003). The lifetime required for the $\mathrm{H}$ II region to expand to its observed size is proportional to $x_{\mathrm{HI}}^{-1 / 3}$. The geometric determination of the neutral fraction can therefore be directly used to determine also the quasar lifetime measured from the combination of $R_{p}$ and $x_{\mathrm{H}}$. Thus, Ly $\alpha$ and redshifted $21 \mathrm{~cm}$ measurements of the expanding $\mathrm{H}$ II regions around the highest redshift quasars will allow a direct determination of quasar ages on a case by case basis and constrain quasar formation models (e.g., Haiman \& Loeb 2001).

On the basis of current number counts of bright highredshift quasars and the preliminary design of LOFAR, we forecast that each LOFAR field (of radius $\sim 11^{\circ}$ ) will contain $\sim 2$ active quasars as bright as the observed SDSS quasars within one bandpass of frequency $(\sim 4 \mathrm{MHz})$. An integration time of $\sim 100 \mathrm{hr}$ should be sufficient to detect the rings of redshifted $21 \mathrm{~cm}$ emission from the warm IGM outside the quasar $\mathrm{H}$ II regions, so long as $x_{\mathrm{H}}$ is on the order of unity. Smaller neutral fractions will result in the $\mathrm{H}$ II regions being much more difficult to detect. In the presence of a strong cosmic Ly $\alpha$ background, these rings should remain visible after the quasar has turned off. This would result in the detection of a large number of fossil $\mathrm{H}$ in regions that exceeds the number of active quasars by the inverse of the quasar duty cycle $(\sim 100)$.

Arcminute observations of quasar Strömgren spheres will provide an alternative method for measuring the spectral step that makes the so-called global signature of reionization (Shaver et al. 1999). The brightness temperature measured in the spectral range corresponding to the ionized gas within the $\mathrm{H}$ II region provides a calibration of the foreground emission at the same redshift. This calibration will help overcome the difficulties associated with measuring the global signature of an extended reionization epoch (as suggested by the large electron scattering optical depth measured by WMAP; Kogut et al. 2003) on top of a frequency dependent foreground. Detection of the signal for a statistical sample of quasars would help calibrate the expected scatter in the reionization redshift of different regions in the universe (Barkana \& Loeb 2004b). 
Finally, we emphasize that the redshifted $21 \mathrm{~cm}$ observation of existing SDSS quasars will determine whether the IGM was significantly neutral at $z>6.3$ as suggested by their observed Ly $\alpha$ spectra (Wyithe \& Loeb 2004). When combined with the high value of the optical depth for electron scattering measured by WMAP (Kogut et al. 2003), a significant neutral fraction at $z \sim 6.3$ would imply a complex reionization history and possibly an early reionization phase driven by PopulationIII stars.

This work was supported in part by NASA grant NAG 513292, and by NSF grants AST-0071019 and AST-0204514 (for A. L.).
Barkana, R., \& Loeb, A. 2004a, ApJ, 601, 64 2004b, ApJ, in press (astro-ph/0310338)

Burke, B. F., \& Graham-Smith, F. 1997, An Introduction to Radio Astronomy (Cambridge: Cambridge Univ. Press)

Cen, R. 2003, ApJ, 591, 12

Cen, R., \& Haiman, Z. 2000, ApJ, 542, L75

Chen, X., \& Miralda-Escudé, J. 2004, ApJ, 602, 1

Ciardi, B., \& Madau, P. 2003, ApJ, 596, 1

Di Matteo, T., Perna, R., Abel, T., \& Rees, M. J. 2002, ApJ, 564, 576

Elvis, M., et al. 1994, ApJS, 95, 1

Fan, X., et al. 2001, AJ, 122, 2833 2003, AJ, 125, 1649

Field, G. B. 1958, Proc. IRE, 46, 240

Furlanetto, S., \& Loeb, A. 2003, ApJ, submitted

Furlanetto, S., Sokasian, A., \& Hernquist, L. 2004, MNRAS, 347, 187

Gnedin, N. Y., \& Ostriker, J. P. 1997, ApJ, 486, 581

Gnedin, N., \& Shaver, P. A. 2003, ApJ, submitted (astro-ph/0312005)

Gunn, J. E., \& Peterson, B. A. 1965, ApJ, 142, 1633

Haiman, Z., \& Hui, L. 2001, ApJ, 547, 27

Haiman, Z., \& Loeb, A. 2001, ApJ, 552, 459

Iliev, I. T., Scannapieco, E., Martel, H., \& Shapiro, P. R. 2003, MNRAS, 341,81

Iliev, I. T., Shapiro, P. R., Ferrara, A., \& Martel, H. 2002, ApJ, 572, L123

Jakobsen, P., Jansen, R., A., Wagner, S., \& Reimers, D. 2003, A\&A, 397, 891

Kogut, A., et al. 2003, ApJS, 148, 161

Loeb, A., \& Rybicki, G. B. 1999, ApJ, 524, 527

Loeb, A., \& Zaldarriaga, M. 2003, Phys. Rev. Lett., submitted (astro$\mathrm{ph} / 0312134)$

\section{REFERENCES}

Madau, P., Meiksin, A., \& Rees, M. J. 1997, ApJ, 475, 429

Madau, P., \& Rees, M. J. 2000, ApJ, 542, L69

Martini, P. 2003, preprint (astro-ph/0304009)

Martini, P., \& Weinberg, D. H. 2001, ApJ, 547, 12

Oh, S. P., \& Mack, K. J. 2003, MNRAS, 346, 871

Richards, G. T., Vanden Berk, D. E., Reichard, T. A., Hall, P. B., Schneider,

D. P., SubbaRao, M., Thakar, A. R., \& York, D. G. 2002, AJ, 124, 1

Rybicki, G. B., \& Lightman, A. P. 1979, Radiative Processes in Astrophysics (New York: Wiley), 288

Scott, D., \& Rees, M. J. 1990, MNRAS, 247, 510

Shaver, P. A., Windhorst, R. A., Madau, P., \& de Bruyn, A. G. 1999, A\&A, 345,380

Shull, J. M., \& van Steenberg, M. E. 1985, ApJ, 298, 268

Spergel, D. N., et al. 2003, ApJS, 148, 175

Telfer, R. C., Zheng, W., Kriss, G. A., \& Davidsen, A. F. 2002, ApJ, 565, 773

Tozzi, P., Madau, P., Meiksin, A., \& Rees, M. J. 2000, ApJ, 528, 597

Vanden Berk, D. E., et al. 2001, AJ, 122, 549

White, R. L., Becker, R. H., Fan, X., \& Strauss, M. A. 2003, AJ, 126, 1

Wouthuysen, S. A. 1952, AJ, 57, 31

Wyithe, J. S. B., \& Loeb, A. 2003a, ApJ, 586, 693 2003b, ApJ, 595, 614

2004, Nature, 427, 815

Yu, Q., \& Tremaine, S. 2002, MNRAS, 335, 965

Yuan, W., Brinkmann, W., Siebert, J., \& Voges, W. 1998, A\&A, 330, 108

Zaldarriaga, M., Furlanetto, S., \& Hernquist, L. 2004, ApJ, in press (astro-ph/0311514) 